\title{
Bardet Biedl syndrome in South Africa: A single founder mutation
}

\author{
K Fieggen, ${ }^{1} \mathrm{MB} \mathrm{ChB}$, FCPaed, Cert Med Genet; C Milligan, ${ }^{1} \mathrm{MB} \mathrm{ChB}$; B Henderson, ${ }^{2} \mathrm{MB} \mathrm{ChB}, \mathrm{MMed}(\mathrm{Paed}), \mathrm{DCH} ;$ \\ A I Esterhuizen, ${ }^{3,4} \mathrm{MSc}(\mathrm{Med})$ \\ ${ }^{1}$ Division of Human Genetics, Department of Medicine, University of Cape Town, South Africa \\ ${ }^{2}$ Division of Clinical Genetics, Department of Neurology, University of the Free State, Bloemfontein, South Africa \\ ${ }^{3}$ Division of Human Genetics, Department of Pathology, University of Cape Town, South Africa \\ ${ }^{4}$ National Health Laboratory Service, Groote Schuur Hospital, Cape Town, South Africa
}

Corresponding author: K Fieggen (karen.fieggen@uct.ac.za)

\begin{abstract}
Background. Bardet Biedl syndrome (BBS) is a multisystem disorder characterised by obesity, polydactyly, intellectual disability and loss of vision due to a progressive retinopathy. Although typically a highly heterogeneous autosomal recessive disease, homozygosity for single mutation in BBS 10 has been identified in a significant number of affected individuals tested in South Africa (SA).

Objectives. To delineate the ethnic distribution and clinical phenotype in a cohort of SA BBS patients with the K243IfsX15 mutation in BBS 10 and discuss the implications for genetic testing of and counselling for this disorder in SA.

Method. This was a descriptive cross-sectional study collating clinical and laboratory data retrospectively in a genetically homogenous subgroup of BBS patients from SA.

Results. A total of 76 patients from 74 families were tested. Homozygosity for the K243IfsX15 BBS 10 mutation was found in 50 families (67\%) and heterozygosity for the same mutation in an additional two affected individuals. With the exception of one patient of mixed ancestry, all were black South Africans from different language groups. This is in keeping with the observation that BBS is more common in this ethnic group compared with white and coloured patients in SA, first made by Prof. Beighton nearly 3 decades ago. A subset of 15 patients available for detailed phenotyping confirmed consistency with well-described features of the disorder, with some overlap with other ciliopathies. The onset of visual impairment was early in our cohort, before the age of 8 years, cognitive impairment was significant, and renal and cardiac abnormalities were infrequently encountered.

Conclusion. The high frequency of homozygosity for a single mutation in an ethnic subset of the SA population is strongly suggestive of a founder effect. This has allowed establishment of a diagnostic test with a high yield in our local population. Better understanding of the phenotype will improve earlier recognition of the disorder to allow for appropriate intervention. Testing can confirm but not negate a clinical diagnosis, and can permit carrier and prenatal testing in informative families.
\end{abstract}

S Afr Med J 2016;106(6 Suppl 1):S72-S74. DOI:10.7196/SAMJ.2016.v106i6.11000

To Prof. Peter Beighton, 'Do genetics and see the world.'... We may not yet have seen the world, but we have seen and learnt so much through your attention to detail, critical thinking, complex problem solving and academic rigour. Your boundless energy and enthusiasm for medical genetics and life we will always treasure.

Bardet Biedel syndrome (BBS) (MIM 209900) is a multisystem disorder resulting from disruption of cilial function. The most consistent clinical features include a progressive rod cone retinal dystrophy, obesity, polydactyly and developmental delay/ cognitive impairment. ${ }^{[1]}$ Other frequent associations are urogenital malformations, hypogonadism, and both structural and functional renal abnormalities. ${ }^{[1-3]}$ Diagnostic criteria have been established with these findings listed as primary features ${ }^{[3]} \mathrm{A}$ characteristic facial phenotype comprising macrobrachycephaly, with short, narrow palpebral fissures, a long, shallow philtrum with thin upper lip and downturned corners of the mouth is described. ${ }^{[3,4]}$ The clinical spectrum shows overlap with a number of other genetic disorders involving abnormalities of ciliogenesis, such as McKusick-Kaufman syndrome and Alström syndrome. ${ }^{[5]}$ Additional reports have expanded both the ocular and systemic phenotype. ${ }^{[6-9]}$

BBS is typically an autosomal recessive disorder. Although oligogenic inheritance is described, it is a highly heterogeneous condition and to date 21 genes (BBS 1-19, NPHP1 and IFT172) that account for more than $80 \%$ of affected individuals have been implicated. ${ }^{[10]}$ Most of the genes code for proteins belonging to two functional groups. $B B S 1,2,4,5,7,8$ and 9 are responsible for the formation of a complex known as the BBSome, important in promoting ciliogenesis. BBS 6,10 and 12 code for type II chaperonins, which contribute to assembly of the BBSome. ${ }^{[11]}$ The contribution of these genes to the mutational burden in BBS varies between populations. BBS 10 is the major contributor explaining $20 \%$ of cases and, together with BBS 1,2 and 4 account for $\sim 60 \%$ of mutations worldwide. ${ }^{[12,13]}$ More than $80 \%$ of disease-causing alleles are private mutations with, to date, only two mutations, BBS 1 M390R and BBS 10 C91Lfs X5, accounting for $>5 \%$ of cases. ${ }^{[12]}$

The observation that BBS is one of the more frequently encountered autosomal recessive disorders in the indigenous black population of South Africa (SA) was first made in 1986 by Beighton and Botha. ${ }^{[14]}$ In a small collaborative study with the Héon laboratory at The Hospital for Sick Children in Toronto in which seven SA BBS patients underwent multigene testing, all four black SA BBS patients included were found to be homozygous for a $4 \mathrm{bp}$

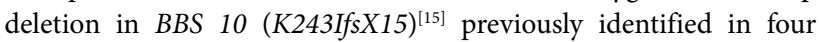
Middle Eastern families. ${ }^{[13,16]}$ Different mutations were identified in the remaining three coloured patients. Subsequent investigation 
showed that an additional $6 / 7$ patients tested carried the same mutation. This, together with the finding of a shared haplotype in the original cohort, strongly suggested a founder mutation. ${ }^{[15]}$ Testing for this specific mutation was made diagnostically available through the National Health Laboratory Service at Groote Schuur Hospital in 2009.

This study was undertaken to delineate the detection rate and clinical phenotype of a subgroup of BBS patients homozygous for the K243IfsX15 mutation.

\section{Methods}

A total of 84 South Africans from 74 BBS families underwent molecular genetic testing between January 2007 and June 2014. In eight samples, the indication for testing was for carrier status and these were excluded from further analysis. Samples were sent from many parts of SA, including the Western Cape, the Eastern Cape, Gauteng, Limpopo, the Free State and KwaZulu-Natal provinces.

\section{Results}

Of the patient samples tested for the K243IfsX15 mutation in BBS 10, homozygosity was found in 50 families (67\%) and heterozygosity in a further 2 families. In the remaining 22 families testing negative for this single mutation, 3 coloured patients have subsequently had causative mutations in other BBS genes identified: 1 with compound heterozygosity in BBS 2 and 2 brothers heterozygous for novel BBS 10 mutations predicted to be disease causing. ${ }^{[6]}$ From the limited information available, a number of patients testing negative did not have more than one or two primary diagnostic criteria, suggesting that the diagnosis may be uncertain. Ethnicity was not documented for all patients, but where available, confirmed that all further positive analyses were found in patients of indigenous black SA descent, with white and coloured patients accounting for a significant number of negative analyses.

There was no documented consanguinity in any of the families. At the time of analysis, there was a wide distribution of ages, from 2 months to 42 years, with the majority of the cohort in the 1st and 2nd decade of life. Gender distribution showed a significant predominance of males. Further analysis of the clinical phenotype was confined to 15 patients homozygous for the K243Ifs X15 BBS10 mutation who were available for comprehensive phenotyping by one or more of the authors. Findings were typical of those well described in BBS, but of note is that all patients were obese, all but one had polydactyly involving at least three

Table 1. The spectrum of clinical findings in a subcohort of BBS patients homozygous for the K243IfsX15 mutation in BBS $10(n=15)^{\star}$

\begin{tabular}{|c|c|c|}
\hline Clinical features & $n(\%)$ & Additional comments \\
\hline Obesity & $15(100)$ & \\
\hline Typical facies & $15(100)$ & $\begin{array}{l}\text { Macrobrachycephaly, deep-set eyes, } \\
\text { almond-shaped palpebral fissures, large } \\
\text { ears, low nasal bridge with broad nasal root } \\
\text { and anteverted nares }\end{array}$ \\
\hline Polydactyly & $14(93)$ & Type A postaxial (three or four limbs) \\
\hline Eyes & & Pigmentary retinopathy present by the age \\
\hline Visual disability & $14(93)$ & \\
\hline Nystagmus & $10(66)$ & \\
\hline \multicolumn{3}{|l|}{$\begin{array}{l}\text { Cognitive impairment/ } \\
\text { developmental delay }\end{array}$} \\
\hline Severe & $1(7)$ & \\
\hline Moderate & $8(53)$ & \\
\hline Mild & $6(40)$ & \\
\hline Renal & & Infantile cystic kidneys/unilateral \\
\hline Structural abnormality & $2(13)$ & hydronephrosis \\
\hline Renal failure & $1(7)$ & \\
\hline Enuresis & $6(40)$ & \\
\hline \multicolumn{3}{|l|}{ Genital } \\
\hline Male $(n=10)$ & $10(100)$ & Small/undescended testes, micropenis \\
\hline Female $(n=5)$ & $1(20)$ & Vaginal atresia, hydrometacolpos \\
\hline Hepatic & $8(53)$ & Elevated transaminases \\
\hline \multicolumn{3}{|l|}{ Cardiovascular } \\
\hline Congenital heart disease / murmur & $0(0)$ & \\
\hline Hypertension & $2(13)$ & \\
\hline \multicolumn{3}{|l|}{ Endocrine } \\
\hline Diabetes mellitus & $2(13)$ & \\
\hline Hypothyroidism & $1(7)$ & \\
\hline
\end{tabular}

limbs and all male patients had evidence of hypogonadism. Visual impairment began with poor night vision, but by the age of 8 years, parents had noticed reduced daytime visual acuity interfering with function in their affected children. Cognitive impairment was considered moderate or severe in over half of the group. In some of the patients, this had been formally assessed by documented Developmental Quotient or Intelligence Quotient scores, but in others the severity was assigned on the basis of functional abilities. In contrast, renal and cardiovascular abnormalities were infrequent. The spectrum of clinical findings in this subcohort is represented in Table 1.

\section{Discussion}

BBS is a complex disorder encountered in our local population, resulting in significant disability for those affected. This study provides confirmation that homozygosity for the K243IfsX15 mutation in BBS 10 is a major contributor to the genotype of SA BBS patients. Although the majority of patients tested were isiXhosa speakers, the mutation was also found in BBS patients of Sotho and Venda ancestry, suggestive of a founder mutation occurring early in the migration of bantu language speakers of southern Africa.

The phenotypic expression in this cohort of BBS patients reflects the pleiotropic effects of cilial dysfunction well described in BBS. ${ }^{[2,3]}$ Both intra- and interfamilial variability is well recognised, but some limited genotype-phenotype correlations are emerging. ${ }^{[15,17]}$ The most frequently encountered manifestations were polydactyly, obesity, visual disturbance, cognitive impairment and male hypogonadism, which 
occurred in $>90 \%$ of patients. There is no clear explanation for the male predominance in patient samples, but it is possible that that early detection of hypogenitalism in boys may prompt testing more readily.

Visual disturbance had an early onset in most of our cohort, with functional visual disability both at night and in the day present by the age of 8 years in the large majority, and all older patients examined were legally blind by mid-adolescence. This is in keeping with previous reports suggesting that mutations in the chaperonin genes associated with BBS may have an earlier onset of retinopathy. ${ }^{[15]}$ Developmental delay/cognitive impairment occurred in the whole cohort and was more severe than described in more heterogeneous cohorts, where intellectual disability occurs in about two-thirds of cases and is usually mild in up to $77 \%$ of cases. ${ }^{[1,2]}$ All but one of our cohort had type A postaxial polydactyly present in at least three limbs, which could suggest a high penetrance for digital abnormalities with this mutation.

In contrast with the reported literature, which indicates that renal abnormalities and altered renal function occur in $>50 \%$ of individuals with BBS, structural renal abnormalities and disorders of renal function as reflected by elevated serum creatinine levels were infrequent in our patients. ${ }^{[8,9]}$ An age-related evolution of the phenotype is well documented in $\mathrm{BBS},{ }^{[1,6]}$ and the younger age distribution of our cohort may have influenced this. Primary enuresis was common into late childhood and adolescence in our cohort, and likely reflects the recognised urinary concentration deficits that occur in BBS. ${ }^{[8]}$ Prospective studies have shown cardiovascular abnormalities in up to $52 \%$ of BBS patients ${ }^{[9]}$ and an incidence of congenital heart disease in BBS of $7-19 \%{ }^{[1,7]}$ With the exception of two patients with hypertension, clinically significant cardiovascular abnormalities were not encountered in our cohort. The clinical finding in this subgroup of patients homozygous for the K243IfsX15 mutation is in keeping with those described in four Omani patients with the same genotype. ${ }^{[16]}$

Genetic testing for highly heterogeneous disorders is not always routinely available. A stratified approach of testing in BBS, prioritising mutations and genes with greatest frequency, has been suggested, ${ }^{[15]}$ although next generation sequencing panels may be a better strategy in more heterogeneous populations. ${ }^{[10]}$ In a resource-constrained SA setting with limited access to multigene panels, first-line testing for the K243IfsX15 mutation in black South Africans with features of BBS is recommended and can be expected to provide a high diagnostic yield. Families with a positive genetic diagnosis can then be offered the option of prenatal diagnosis and carrier testing of the at-risk relatives. Genetic counselling is recommended as the limitations and implications of both positive and negative results needs to be carefully communicated.

\section{Conclusion}

A single BBS 10 mutation (K243IfsX15) accounted for $68 \%$ of diseasecausing alleles in this study of SA BBS patients. Genetic testing for this single mutation is simple and cost effective, and allows for confirmatory testing in a significant proportion of SA patients in whom a diagnosis of BBS is suspected. It is, however, essential that the limitations of testing for a single mutation in a condition with such marked genetic heterogeneity be clearly understood and communicated when testing is considered and results delivered. ${ }^{[18]}$

Knowledge of the phenotypic spectrum and age-related penetrance of BBS will improve diagnostic accuracy and allow for earlier diagnosis. In younger children, a diagnosis of BBS should be considered when polydactyly and genital abnormalities are encountered, particularly when accompanied by the characteristic facial features, obesity and developmental delay. Older children may come to attention when presenting for evaluation of obesity, or reduced night vision or poor visual acuity. A prompt diagnosis will allow for appropriate care for the individual and genetic counselling for the family.

Acknowledgements. We wish to thank colleagues who performed the initial analysis in the Héon laboratory as well as colleagues who referred patients for testing and provided clinical information, in particular Ms Carol Massyn and the University of Cape Town Medical Genetic team. We would also like to pay tribute to the patients and their families who live with this challenging disorder and continue to inspire us with their strength and courage.

\section{References}

1. Forsythe E, Beales PL. Bardet-Biedl syndrome. Eur J Hum Genet 2013;21(1):8-13. DOI:10.1038/ ejhg. 2012.115

2. Deveault C, Billingsley G, Duncan IL, et al. BBS genotype-phenotype assessment of a multiethnic patient

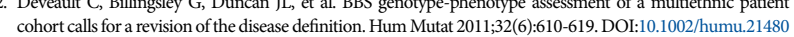
cohort calls for a revision of the disease definition. Hum Mutat 2011;32(6):610-619. DOl:10.1002/humu.21480
3. Beales PL, Elcioglu N, Woolf AS, et al. New criteria for improved diagnosis of Bardet-Biedl syndrome: 3. Beales PL, Elcioglu N, Woolf AS, et al. New criteria for improved
Results of a population survey. J Med Genet 1999;36(6):437-446.

4. Lorda-Sanchez I, Ayuso C, Sanz R, Ibañez A. Does Bardet-Biedl syndrome have a characteristic face? J Med Genet 2001;38(5):E14.

5. Schaefer E, Durand M, Stoetzel C, et al. Molecular diagnosis reveals genetic heterogeneity for the overlapping MKKS and BBS phenotypes. Eur J Med Genet 2011;54(2):157-160. DOI:10.1016/j. ejmg.2010.10.004

6. Daniels AB, Sandberg MA, Chen J, Weigel-DiFranco C, Fielding Hejtmancic J, Berson EL. Genotypephenotype correlations in Bardet-Biedl syndrome. Arch Ophthalmol 2012;130(7):901-907. DOI:10.1001/archophthalmol.2012.89

7. Moore SJ, Green JS, Fan Y, et al. Clinical and genetic epidemiology of Bardet-Biedl syndrome in Newfoundland: A 22-year prospective, population-based, cohort study. Am J Med Genet A 2005;132A(4):352-360. DOI:10.1002/ajmg.a.30406

8. Putoux A, Attie-Bitach T, Martinovic J, Gubler MC. Phenotypic variability of Bardet-Biedl syndrome: 8. Putoux A, Attie-Bitach T, Martinovic J, Gubler MC. Phenotypic variability of Bardet-Biedl synd
Focusing on the kidney. Pediatr Nephrol 2012;27(1):7-15. DOI:10.1007/s00467-010-1751-3

9. Imhoff O, Marion V, Stoetzel C, et al. Bardet-Biedl syndrome: A study of the renal and Imhoff O, Marion V, Stoetzel C, et al. Bardet-Biedl syndrome: A study of the renal and
cardiovascular phenotypes in a French cohort. Clin J Am Soc Nephrol 2011;6(1):22-29. cardiovascular phenotypes in
DOI:10.2215/CJN.03320410

10. Ece Solmaz A, Onay H, Atik T, et al. Targeted multi-gene panel testing for the diagnosis of Bardet Biedl syndrome: Identification of nine novel mutations across $B B S 1, B B S 2, B B S 4, B B S 7, B B S 9, B B S 10$ genes. Eur J Med Genet 2015;58(12):689-694. DOI:10.1016/j.ejmg.2015.10.011

11. Seo S, Baye LM, Schulz NP, Beck JS, et al. BBS6, BBS10, and BBS12 form a complex with CCT/TRiC family chaperonins and mediate BBSome assembly. Proc Natl Acad Sci U S A 2010;107(4):1488-1493. DOI:10.1073/pnas.0910268107

12. Billingsley G, Deveault C, Héon E. BBS mutational analysis: a strategic approach. Ophthalmic Genet 2011;32(3):181-187. DOI:10.3109/13816810.2011.567319

13. Stoetzel C, Laurier V, Davis EE, et al. BBS10 encodes a vertebrate-specific chaperonin-like protein and is a major BBS locus. Nat Genet 2006;38(5):521-524. DOI:10.1038/ng1771

14. Beighton P, Botha MC. Inherited disorders in the black population of Southern Africa: Part II. Gene Beighton P, Botha MC. Inherited disorders
disorders. S Afr Med J 1986;69(5):293-296.

15. Billingsley G, Bin J, Fieggen KJ, et al. Mutations in chaperonin-like BBS genes are a major contributor . Billingsley G, Bin J, Fieggen KJ, et al. Mutations in chaperonin-like BBS genes are a major contributor
to disease development in a multiethnic Bardet-Biedl syndrome patient population. J Med Genet 2010;47(7):453-463. DOI:10.1136/jmg.2009.073205

16. White DR, Ganesh A, Nishimura D, et al. Autozygosity mapping of Bardet-Biedl syndrome to $12 q 21.2$ and confirmation of FLJ23560 as BBS10. Eur J Hum Genet 2007;15(2):173-178. DOI:10.1038/ sj.ejhg. 5201736

17. Castro-Sánchez S, Álvarez-Satta M, Cortón M, Guillén E, Ayuso C, Valverde D. Exploring genotypephenotype relationships in Bardet-Biedl syndrome families. J Med Genet 2015;52(8):503-513. DOI:10.1136/jmedgenet-2015-103099

18. Sapp JC, Nishimura D, Johnston JJ, et al. Recurrence risks for Bardet-Biedl syndrome: Implications of locus heterogeneity. Genet Med 2010;12(10):623-627. DOI:10.1097/GIM.0b013e3181f07572 